\title{
Producción Divergente Explicativa: La relación entre resiliencia y creatividad
}

\author{
Óscar Sánchez Hernández ${ }^{1}$, Francisco Xavier Méndez ${ }^{1}$ \\ $\stackrel{\text { y }}{\text { Judy Garber }}{ }^{2}$ \\ ${ }^{1}$ Departamento de Personalidad, Evaluación y Tratamiento Psicológicos. \\ Universidad de Murcia \\ ${ }^{2}$ Vanderbilt University, Nashville (EE.UU.)
}

España / EEUU

Correspondencia:Óscar Sánchez Hernández. Facultad de Psicología. Campus Universitario de Espinardo (Murcia). CP: 30100. España. E-mail: oscarsh@um.es

() Education \& Psychology I+D+i and Ilustre Colegio Oficial de la Psicología de Andaluacía Oriental (Spain) 


\section{Resumen}

Introducción. El objetivo del estudio es describir y analizar un nuevo test y constructo, la Producción Divergente Explicativa (PDE), definido como la capacidad de observar las situaciones adversas desde varios puntos de vista. A nivel teórico supone un puente entre el modelo reformulado de indefensión aprendida como modelo de resiliencia, con la inteligencia creativa. En este trabajo se estudia la relación de la PDE con la inteligencia creativa, así como las diferencias de género.

Método. La muestra estuvo formada por 89 participantes $1^{\circ}$ y $2^{\circ}$ de ESO pertenecientes a siete centros educativos de la Región de Murcia a la que se les aplicó dos test para medir la relación entre PDE y creatividad. Se realizaron análisis correlacionales, de regresión y de diferencia de medias independientes respecto al género y baja o alta PDE.

Resultados. Los distintos análisis estadísticos que se aplicaron reflejan que los participantes que produjeron más explicaciones causales en general y más explicaciones causales resilientes en particular, ante hipotéticas adversidades, se asociaron con una mayor inteligencia creativa. No se hallaron diferencias significativas de género.

Discusión. Siendo unos resultados interesantes, al relacionar el estilo explicativo resiliente con la creatividad, es necesario nuevos estudios, con mayores muestras, estudiar la relación con otras variables afines (flexibilidad cognitiva, flexibilidad explicativa, optimismo disposicional, curiosidad, etc) así como estudiar su papel en las hipótesis de los mediadores sobre la mejora en intervenciones preventivas y de tratamiento de problemas emocionales.

Palabras Clave. Producción divergente explicativa, estilo explicativo, creatividad, resiliencia, optimismo, género. 


\title{
Diverse Explanatory Production (DEP): the relationship between resilience and creativity
}

\begin{abstract}
Introduction. The aim of the study is to describe and analyze a new test and construct Diverse Explanatory Production (DEP), defined as the ability to observe adverse situations from various points of view. At the theoretical level is a bridge between the reformulated model of learned helplessness as a model of resilience, with creative intelligence. In this paper the relationship between DEP with creative intelligence, as well as gender differences are studied.
\end{abstract}

Method. The sample consisted of 89 participants 1st and 2nd of ESO from seven schools in the Region of Murcia which were applied two tests to measure the relationship between DEP and creativity. Correlational analysis, regression and difference of independent means on gender and low or high DEP were performed.

Results. The various statistical analyzes were applied to reflect participants produced more causal explanations in general and more resilient causal explanations in particular regarding hypothetical adversities associated with greater creative intelligence. No significant gender differences were found.

Discussion. Being interesting results, by relating the resilient explanatory style with creativity, new studies, with larger samples is necessary to study the relationship with other related variables (cognitive flexibility, flexibility explanatory, dispositional optimism, curiosity etc) as well as studying its role in mediators assumptions about improving preventive interventions and treatment of emotional problems.

Keywords: Diverse Explanatory Production, explanatory style, creativity, resilience, optimism, gender.

Reception: 10.24.15 Initial acceptance: 10.27.15 Final acceptance: 11.19 .15 


\section{Introducción}

El estudio y promoción de la resiliencia en la infancia y adolescencia puede ayudar a los jóvenes a superar adversidades que tendrán que afrontar en sus vidas, bien sean consecuencias del estrés diario (académico, social...), de las crisis vitales y/o desastres naturales. El estilo explicativo es una de las variables más investigadas en torno a la resiliencia (Seligman, 2005). Las personas con un estilo explicativo resiliente u optimista tiende a explicar las adversidades con atribuciones externas, temporales y específicas; por el contrario, el estilo explicativo pesimista realiza atribuciones internas, permanentes y globales de los eventos negativos. La forma de explicar los acontecimientos influye en la motivación para perseverar en las metas propuestas y en la adaptación a las adversidades.

Un estilo explicativo resiliente se asocia a índices más bajos de enfermedad física, depresión y suicidio, y -a niveles más elevados- de rendimiento académico y deportivo, adaptación profesional y calidad de vida familiar (Seligman, 1998). Por otra parte, se ha barajado que, entre otras variables cognitivas, un estilo explicativo pesimista podría ser uno de los factores responsables del incremento de la depresión durante la adolescencia. La investigación también indica que gran parte de este aumento durante la adolescencia se explica por un aumento de la depresión entre las niñas en particular. Se ha encontrado que un estilo cognitivo negativo mayor en las chicas adolescentes mediaba las diferencias de género en sintomatología depresiva (Hankin y Abramson, 2002).

También se ha hallado que los niños y adolescentes que tienden a interpretar las situaciones sociales con el primer pensamiento pesimista que les pasa por la cabeza, tienden a reaccionar de forma hostil ante estas situaciones. Por ello, se les enseña, primero a pararse a pensar antes de reaccionar, e intentar encontrar otras posibles causas que hayan producido la situación lo que, en primer lugar, les alivia a nivel emocional y, por otra parte, les ayuda a encontrar otras formas de afrontar la situación sin caer en la pasividad o la agresividad (Seligman et al, 2005).

El interés en este nuevo constructo surge de la observación de una de las técnicas del programa preventivo de Seligman et al. (2005), basado en la promoción del estilo explicativo resiliente, para ayudar a los niños que tienden a ver su contribución a los problemas en términos de todo o nada y tienen dificultad para asumir su parte de responsabilidad. Dicha 
técnica consistente en proponer el mayor número de causas que podrían explicar el por qué se ha producido cierta adversidad, valorando el problema con la mayor objetividad posible, teniendo en cuenta tanto aquellas que dependen de uno mismo y de las que puede responsabilizarse como otras que dependen de los demás o de otros factores externos a la persona.

La producción divergente explicativa podría relacionarse con modelos de solución de problemas, así Guilford en 1977 afirmaba que "en la resolución de problemas y el pensamiento creativo encontramos habilidades intelectuales que funcionan conjuntamente...". Destacamos el programa de Mahoney (1981), denominado SCIENCE, que propone como paso previo a la búsqueda de soluciones, el identificar las causas del problema. Las explicaciones sobre las posibles causas que han producido un determinado problema podrían determinar el marco psicológico dentro del que se van a encontrar las posibles soluciones al mismo. La producción divergente explicativa se contextualiza también dentro del constructo de flexibilidad psicológica, que entre otras características está relacionado con la capacidad de observar los problemas desde varios puntos de vista (Kashdan y Rottenberg, 2010).

\section{Objetivo e hipótesiss}

Aunque se le ha concedido importancia a evaluar el estilo explicativo y su relación con otras variables como la depresión, se pone de relieve la falta de un instrumento de medida que evalúe esta variable referida a la habilidad de generar diversas y variadas explicaciones causales acerca de las adversidades. Este nuevo test, que se presenta, por primera vez, en este informe de investigación, pretende evaluar el constructo que hemos nombrado como producción divergente explicativa (PDE) definida como la capacidad de producir el mayor número de causas o atribuciones posibles para explicar unas hipotéticas adversidades. Al igual que el test CREA (Corbalán et al, 2003), que mide producción divergente, la PDE podría estar relacionada con la flexibilidad, la fluidez o la originalidad (Guilford, 1977; Torrance, 1974), la búsqueda de problemas, problem finding (Runco, 1994) y el pensamiento lateral, lateral thinking (De Bono, 1998).

El objetivo de esta investigación es analizar la relación de la PDE con la inteligencia creativa, así como las diferencias de género. La hipótesis es que la relación de una mayor PDE (mayor número de explicaciones causales ante hipotéticas adversidades), en general, y de una mayor PDE resiliente (mayor número de explicaciones causales resilientes, definidas en este estudio como temporales y específicas), en particular, con la creatividad es positiva. 
Respecto a las diferencias de género se esperan mayores puntuaciones de PDE en la muestra de chicos, dado que esta variable podría ser mediadora de las diferencias de género en los problemas emocionales a estas edades.

\section{Método}

\section{Participantes}

Los participantes se reclutaron dentro de una muestra de 1.212 adolescentes de $1^{\circ}$ y $2^{\circ}$ de ESO pertenecientes a siete centros educativos de la Región de Murcia (España). Todos los centros se hallaban ubicados en la ciudad de Murcia, excepto uno situado en la localidad de Abanilla. La muestra final estuvo formada por 89 adolescentes de $1^{\circ}$ y $2^{\circ}$ de ESO pertenecientes a siete centros educativos públicos (80.9\%) y concertados/privados (19.1\%) de la Región de Murcia (España). El porcentaje por género corresponde a un 52\% de chicas y un $48 \%$ de chicos, de edades comprendidas entre los 12 y 15 años (edad media 13,88 años, desviación estándar 0,95).

\section{Instrumentos}

Producción divergente explicativa (PDE). Para la elaboración del test se utilizaron 5 adversidades que los adolescentes han señalado como problemáticas así como en la opinión de expertos (Hankin y Abramson, 2002). Estas son representativas de diversos ámbitos de la vida de los adolescentes (relaciones interpersonales, familiares, académicas y extraescolares): (1) Te gustaría tener una relación sentimental (novio / novia) pero no la tienes; (2) Se organiza una fiesta a la que te gustaría asistir pero nadie te invita; (3) Se reparten las evaluaciones del último trimestre y compruebas que has sacado malas notas; (4) Durante el fin de semana tienes una gran disputa (pelea) con tus padres; (5) Tienes el deseo de formar parte en una actividad extraescolar de ocio (como un equipo de fútbol u otro deporte, un club, recreación, juego...) pero no eres elegido y te quedas fuera.

Las instrucciones que se les dio a los participantes es que intentaran imaginarse con claridad en cada una de las situaciones presentadas y, en dos minutos de tiempo para cada una, escribieran todas las causas por las que creían que podría ocurrirle esa situación. La corrección del test tiene una interpretación cuantitativa (número total de explicaciones causales) y cualitativa, en la que se clasifican el número de explicaciones causales según las dimensiones individuales (personalización, duración y amplitud) del modelo reformulado de indefen- 
sión aprendida (Abramson, Seligman y Teasdale, 1978). Se halló un adecuado acuerdo entre jueces según el coeficiente de correlación intraclase entre las diversas dimensiones: Total atribuciones $(0,94)$, Internas $(0,93)$, Externas $(0,90)$, Permanentes $(0,71)$, Temporales $(0,75)$, Globales (0,86), Específicas (0,78), Permanentes-Globales $(0,81)$, Temporales-Específicas $(0,69)$, Permanentes-Globales-Internas $(0,85)$, Permanentes-Globales-Externas $(0,78)$, Temporales-Específicas-Externas (0,50), Temporales-Específicas-Internas $(0,52)$.

Inteligencia Creativa (CREA; Corbalán et al., 2003). El CREA mide producción divergente. La tarea consiste en la elaboración de preguntas sobre un estímulo determinado. CREA incluye dos laminas destinadas a adolescentes y adultos (A y B) y una para niños (C). En este estudio tan solo se utilizaron las láminas A y B. Se conceden 4 minutos de duración para formular preguntas sobre una lámina que se presenta a los sujetos. El número de preguntas que generen se interpreta como un indicador de flexibilidad cognitiva y como una medida de la capacidad del sujeto para relacionar esquemas cognitivos, algo muy relacionado con la creatividad (Corbalán, Martínez, Donolo, Tejerina y Limiñana, 2003). Es interesante señalar que en la lámina B del CREA se pide a los participantes que hagan preguntas acerca de una situación social, en cambio la lámina A se pide hacer preguntas sobre un objeto inanimado (un teléfono). En otros estudios se han señalado diferencias, respecto a la relación con programas de promoción del bienestar, entre ambas láminas (véase Sánchez-Hernández, 2012) por lo que se analizan de forma separada. La fiabilidad estimada del coeficiente de consistencia interna para las láminas A y B fue de 0,87.

\section{Procedimiento}

A una muestra de 1.212 adolescentes de $1^{\circ}$ y $2^{\circ}$ de ESO pertenecientes a siete centros educativos de la Región de Murcia (España) se les oferto la posibilidad de participar en un proyecto de promoción del bienestar en horario extraescolar accediendo finalmente 89 participantes. En una de las sesiones de este proyecto se realizó la evaluación para este estudio. Primero se pasó la prueba de producción divergente explicativa seguidamente de la medida de creatividad. El test fue corregido por dos colaboradoras entrenadas en clasificar las explicaciones causales en las distintas dimensiones individuales (personalización, duración y amplitud) según el modelo reformulado de indefensión aprendida (Abramson, Seligman y Teasdale, 1978) método ya utilizado anteriormente en otros estudios (Schulman, Castellon, y Seligman, 1989). Se decidió realizar un promedio de las evaluaciones realizadas por ambas expertas para realizar los análisis. 


\section{Análisis de datos}

El grado de asociación entre estas tres variables se analizó mediante el índice de correlación de pearson. Con la finalidad de poder acercarse más a la consideración de una relación predictiva, dado que las correlaciones podrían entenderse en dos sentidos, se decidió hacer análisis de regresión para evaluar el porcentaje de varianza de la prueba de creatividad explicado por la variabe Producción Divergente Explicativa. También se efectuaron contrates de hipótesis sobre medias para muestras independientes con la prueba $t$ de Student tanto para estudiar las diferencias de género como para comparar la puntuación de creatividad entre los participantes con alta PDE con participantes con baja PDE. Para llevar este último análisis se procedió a agrupar a los sujetos en dos grupos (baja o alta PDE) confeccionados a partir de los puntos de corte obtenidos por la media aritmética de las diversas dimensiones de la PDE.

\section{Resultados}

\section{Relaciones correlacionales bivariadas}

A nivel general, los resultados confirmaron que la relación de una mayor PDE en general y de una mayor PDE resiliente (atribuciones temporales y específicas) en particular, con la creatividad, es positiva. Se observa que las atribuciones internas se relacionan más con la creatividad. En la tabla 1 se describen las correlaciones encontradas.

Tabla 1. Correlaciones entre creatividad y producción divergente explicativa junto a la interpretación de la magnitud del tamaño del efecto según Cohen (1988)

\begin{tabular}{ccc}
\hline PRODUCCIÓN DIVERGENTE EXPLICATIVA & CREA A & CREA B \\
\hline Atribuciones totales & $0,43^{* *}$ & $0,32 *$ \\
& $($ media) & $($ media) \\
Atribuciones internas & $0,42^{* *}$ & $0,30^{*}$ \\
& $($ media) & $($ media) \\
Atribuciones externas & 0,21 & 0,18 \\
& $($ baja $)$ & $($ baja) \\
Atribuciones permanentes & 0,18 & 0,07 \\
& $($ baja) & (nula) \\
Atribuciones temporales & $0,49^{* * *}$ & $0,43^{* * *}$ \\
& $($ media) & (media) \\
Atribuciones globales & 0,21 & 0,12 \\
& $($ baja) & (baja) \\
Atribuciones específicas & $0,36^{* *}$ & $0,30^{*}$ \\
& $($ media) & $($ media) \\
& 0 & $-0,05$ \\
Atribuciones permanentes y globales & (nula) & (nula) \\
\hline
\end{tabular}




\begin{tabular}{ccc}
\hline Atribuciones temporales y específicas & $\begin{array}{l}0,42 * * \\
\text { (media) }\end{array}$ & $\begin{array}{c}0,44^{* * *} \\
\text { (media) }\end{array}$ \\
Atribuciones permanentes, globales e internas & 0,01 & $-0,01$ \\
& $($ nula) & (nula) \\
Atribuciones permanentes, globales y externas & 0,02 & $-0,04$ \\
& $($ nula) & (nula) \\
Atribuciones temporales, específicas y externas & 0,21 & $0,33^{* *}$ \\
& $($ baja) & (media) \\
Atribuciones temporales, específicas e internas & $0,41^{* *}$ & $0,37 * *$ \\
& (media) & (media) \\
\hline
\end{tabular}

\section{Relaciones de regresión predictiva}

En los análisis de regresión efectuados, utilizando la PDE como variable predictora, se halló una predicción significativa entre PDE y la creatividad. Concrétamente una mayor PDE en general (atribuciones totales) y una mayor PDE resiliente (atribuciones temporales, específicas) e internas en particular, predice la puntuación en creatividad, tanto en CREA-A como en CREA-B.

Las variables que mejor explican la creatividad, en CREA-A, fueron las atribuciones temporales (AT; 23\% de varianza explicada), atribuciones totales (ATOT; 17\%), atribuciones internas (AI; 16\%), atribuciones temporales y específicas (ATE; 16\%) atribuciones temporales, específicas e internas (ATEI; 15\%) y atribuciones específicas (AE; 11\%). El resto de resultados se puede encontrar en la tabla 2.

Respecto a CREA-B, las variables que mejor la explican fueron las atribuciones temporales y específicas(ATE; 18\%) las atribuciones temporales (AT; 17\%), atribuciones temporales, específicas e internas (ATEI; 12\%), atribuciones temporales, específicas y externas (ATEI; 10\%), atribuciones totales (ATOT; 9\%), atribuciones específicas (AE; 8\%) y atribuciones internas (AI; 7\%),. El resto de resultados se puede encontrar en la tabla 3. 
Tabla 2. Análisis de regresión simple utilizando las distintas dimensiones de la PDE como variables predictoras de CREA A

\begin{tabular}{lllllll} 
CREA-A & $\mathrm{R}$ & $\mathrm{R}^{2}$ & $\mathrm{R}^{2}$ cor. & E. tip. Estimac. & $\mathrm{F}$ & SIG. \\
ATOT & .427 & .183 & .169 & 5.39667 & 13.181 & .001 \\
AI & .424 & .179 & .165 & 5.40729 & 12.898 & .001 \\
AEX & .212 & .045 & .029 & 5.83340 & 2.777 & .101 \\
AP & .178 & .032 & .015 & 5.87406 & 1.925 & .171 \\
AT & .493 & .243 & .230 & 5.19478 & 18.900 & .000 \\
AG & .210 & .044 & .028 & 5.83652 & 2.711 & .105 \\
AE & .361 & .130 & .115 & 5.56699 & 8.832 & .004 \\
APG & .003 & .000 & -.017 & 5.96911 & .000 & .985 \\
ATE & .423 & .179 & .165 & 5.40957 & 12.837 & .001 \\
APGI & .007 & .000 & -.017 & 5.96899 & .003 & .959 \\
APGEX & .022 & .000 & -.016 & 5.96767 & .029 & .866 \\
ATEEX & .209 & .044 & .027 & 5.83752 & 2.690 & .106 \\
ATEI & .410 & .168 & .154 & 5.44321 & 11.952 & .001 \\
\hline
\end{tabular}

CREA-A = Lámina A del test CREA; ATOT = Atribuciones totales; AI = Atribuciones internas; AEX = Atribuciones externas; $\mathrm{AP}=$ Atribuciones permanentes; $\mathrm{AT}=$ Atribuciones temporales; $\mathrm{AG}=$ Atribuciones globales; $\mathrm{AE}=$ Atribuciones específicas; $\mathrm{APG}=$ Atribuciones permanentes y globales; $\mathrm{ATE}=$ Atribuciones temporales $\mathrm{y}$ específicas; APGI = Atribuciones permanentes, globales e internas; APGEX = Atribuciones permanentes, globales y externas; ATEEX = Atribuciones temporales, específicas y externas; ATEI = Atribuciones temporales, específicas e internas.

Tabla 3. Análisis de regresión simple utilizando las distintas dimensiones de la PDE como variables predictoras de CREA B

\begin{tabular}{lllllll} 
CREA-B & $\mathrm{R}$ & $\mathrm{R}^{2}$ & $\mathrm{R}^{2}$ cor. & E. tip. Estimac. & $\mathrm{F}$ & SIG. \\
ATOT & .320 & .103 & .087 & 7.13558 & 6.743 & .012 \\
AI & .301 & .091 & .075 & 7.18311 & 5.876 & .018 \\
AEX & .181 & .033 & .017 & 7.40728 & 2.009 & .162 \\
AP & .067 & .005 & -.012 & 7.51532 & .267 & .607 \\
AT & .434 & .189 & .175 & 6.78521 & 13.708 & .000 \\
AG & .121 & .015 & -.002 & 7.47728 & .872 & .354 \\
AE & .305 & .093 & .078 & 7.17300 & 6.059 & .017 \\
APG & .054 & .003 & -.014 & 7.52117 & .175 & .677 \\
ATE & .438 & .192 & .178 & 6.77044 & 14.026 & .000 \\
APGI & .014 & .000 & -.017 & 7.53158 & .011 & .915 \\
APGEX & .037 & .000 & -.016 & 7.52716 & .081 & .777 \\
ATEEX & .333 & .111 & .096 & 7.10321 & 7.344 & .009 \\
ATEI & .366 & .134 & .119 & 7.00930 & 9.133 & .004 \\
\hline
\end{tabular}

CREA-B = Lámina B del test CREA; ATOT = Atribuciones totales; AI = Atribuciones internas; AEX = Atribuciones externas; $\mathrm{AP}=$ Atribuciones permanentes; $\mathrm{AT}=$ Atribuciones temporales; $\mathrm{AG}=$ Atribuciones globales; $\mathrm{AE}=$ Atribuciones específicas $; \mathrm{APG}=$ Atribuciones permanentes y globales; ATE $=$ Atribuciones temporales $\mathrm{y}$ específicas; APGI = Atribuciones permanentes, globales e internas; APGEX = Atribuciones permanentes, globales y externas; ATEEX = Atribuciones temporales, específicas y externas; ATEI = Atribuciones temporales, específicas e internas. 
Diferencias de género en Producción Divergente Explicativa y en Creatividad

No se encontraron diferencias significativas de género en las variables del estudio. Para una mayor información se puede consultar la Tabla 4.

Tabla 4. Diferencias de medias entre sexo. Descriptivos (media y desviación típica) para cada sexo en las distintas dimensiones de PDE y CREA.

\begin{tabular}{|c|c|c|c|c|c|c|c|c|}
\hline & Género & $\mathrm{n}$ & Media & $\overline{\mathrm{Dt}}$ & Error & $\mathrm{T}$ & g.l. & $P$ \\
\hline \multirow{2}{*}{ CREA-A } & Mujer & 35 & 13,5714 & 5,81219 & ,98244 & \multirow{2}{*}{1,038} & \multirow{2}{*}{61} & \multirow{2}{*}{,304 } \\
\hline & Hombre & 28 & 12,0000 & 6,17042 & 1,16610 & & & \\
\hline \multirow{2}{*}{ CREA-B } & Mujer & 35 & 14,8000 & 7,53736 & 1,27405 & \multirow[t]{2}{*}{1,252} & \multirow[t]{2}{*}{61} & \multirow[t]{2}{*}{,215 } \\
\hline & Hombre & 28 & 12,4643 & 7,12056 & 1,34566 & & & \\
\hline \multirow{2}{*}{ ATOT } & Mujer & 38 & 20,3026 & 8,36623 & 1,35718 & \multirow[t]{2}{*}{,- 784} & \multirow[t]{2}{*}{65} & \multirow[t]{2}{*}{,436 } \\
\hline & Hombre & 29 & 21,8103 & 6,97625 & 1,29546 & & & \\
\hline \multirow{2}{*}{ AI } & Mujer & 38 & 11,5658 & 5,88076 & ,95399 & \multirow[t]{2}{*}{$-1,189$} & \multirow[t]{2}{*}{65} & \multirow[t]{2}{*}{,239 } \\
\hline & Hombre & 29 & 13,2759 & 5,76409 & 1,07036 & & & \\
\hline \multirow{2}{*}{ AEX } & Mujer & 38 & 8,7368 & 5,53946 & ,89862 & \multirow[t]{2}{*}{,202 } & \multirow[t]{2}{*}{65} & \multirow[t]{2}{*}{,841 } \\
\hline & Hombre & 29 & 8,5000 & 3,48978 & 64804 & & & \\
\hline \multirow{2}{*}{ AP } & Mujer & 38 & 11,5526 & 5,27204 & ,85524 & \multirow[t]{2}{*}{,- 262} & \multirow[t]{2}{*}{65} & \multirow[t]{2}{*}{,794 } \\
\hline & Hombre & 29 & 11,8793 & 4,75968 & ,88385 & & & \\
\hline \multirow{2}{*}{ AT } & Mujer & 38 & 8,7368 & 5,14878 & ,83524 & \multirow[t]{2}{*}{,- 997} & \multirow[t]{2}{*}{65} & \multirow[t]{2}{*}{,322 } \\
\hline & Hombre & 29 & 9,9138 & 4,25749 & ,79060 & & & \\
\hline \multirow{2}{*}{$\mathrm{AG}$} & Mujer & 38 & 10,5000 & 6,88221 & 1,11644 & ,022 & 65 & ,983 \\
\hline & Hombre & 29 & 10,4655 & 5,57727 & 1,03567 & & & \\
\hline & Mujer & 38 & 10,0132 & 4,84975 & ,78673 & $-1,039$ & 65 & ,303 \\
\hline AE & Hombre & 29 & 11,3448 & 5,62854 & 1,04519 & & & \\
\hline$\triangle \mathrm{PG}$ & Mujer & 38 & 6,8553 & 5,13587 & ,83315 &,- 249 & 65 & ,804 \\
\hline AI & Hombre & 29 & 7,1552 & 4,50813 & ,83714 & & & \\
\hline & Mujer & 38 & 5,2500 & 3,64608 & 59147 & $-1,242$ & 65 & ,219 \\
\hline AIE & Hombre & 29 & 6,3103 & 3,20550 & ,59525 & & & \\
\hline$\Delta$ & Mujer & 38 & 3,6184 & 3,33579 & ,54114 & $-1,374$ & 65 & , 174 \\
\hline U1 & Hombre & 29 & 4,7759 & 3,52184 & 65399 & & & \\
\hline GEX & Mujer & 38 & 3,1974 & 3,27055 & ,53055 & 1,281 & 65 & ,205 \\
\hline JLA & Hombre & 29 & 2,3103 & 2,04159 & 37911 & & & \\
\hline ATEEX & Mujer & 38 & 2,1579 & 1,88201 & ,30530 & $-1,434$ & 65 & , 156 \\
\hline & Hombre & 29 & 2,7931 & 1,67714 & ,31144 & & & \\
\hline & Mujer & 38 & 2,9868 & 1,95727 & ,31751 & $-1,553$ & 65 &, 125 \\
\hline ATEl & Hombre & 29 & 3,8621 & 2,65887 & ,49374 & & & \\
\hline
\end{tabular}

CREA-A = Lámina A del test CREA; CREA-B = Lámina B del test CREA; ATOT = Atribuciones totales; AI = Atribuciones internas; $\mathrm{AEX}=$ Atribuciones externas; AP = Atribuciones permanentes; AT = Atribuciones temporales; $\mathrm{AG}=$ Atribuciones globales; $\mathrm{AE}=$ Atribuciones específicas; $\mathrm{APG}=$ Atribuciones permanentes y globales; ATE = Atribuciones temporales y específicas; APGI = Atribuciones permanentes, globales e internas; APGEX = Atribuciones permanentes, globales y externas; ATEEX = Atribuciones temporales, específicas y externas; $\mathrm{ATEI}=$ Atribuciones temporales, específicas e internas. 
Diferencia de medias en creatividad entre niveles altos y bajos de Producción Divergente Explicativa.

Se decidió estudiar las diferencias en creatividad según niveles altos o bajos en las distintas dimensiones de PDE utilizando como puntos de corte la media aritmética en las distintas dimensiones comentadas. En general, tanto en CREA-A como en CREA-B, los participantes con una mayor PDE en general, y con más PDE resiliente (atribucione temporales y específicas) mostraron, de forma estadísticamente significativa, mayor creatividad respecto al grupo con menor PDE, en general, y el grupo con menor PDE resiliente en particular. Al comparar los resultados en creatividad del CREA-A también se halló que los participantes con alta PDE en atribuciones internas (AI) mostraron, de forma estadísticamente significativa, mayor creatividad respecto al grupo con menor PDE en AI. Los resultados pueden encontrarse en la tabla 5 y la tabla 6.

Tabla 5. Diferencias de medias en CREA-A según nivel alto o bajo en PDE. Media y desviación típica en CREA-A según nivel alto o bajo en PDE

\begin{tabular}{cccccccc}
\hline $\begin{array}{c}\text { Niveles de } \\
\text { PDE }\end{array}$ & $\mathrm{N}$ & Media & Dt & Error & $\mathrm{T}$ & g.l. & $\mathrm{P}$ \\
\hline ATOT-Alta & 28 & 15,3929 & 6,17246 & 1,16648 & & & \\
ATOT-Baja & 33 & 11,1818 & 5,01532 &, 87305 & & &, 005 \\
\hline AI-Alta & 31 & 15,3548 & 5,82837 & 1,04681 & 3,233 & 59 &, 002 \\
AI-Baja & 30 & 10,8000 & 5,14212 &, 93882 & & & \\
\hline AEX-Alta & 26 & 14,3846 & 6,46267 & 1,26743 & & & \\
AEX-Baja & 35 & 12,1714 & 5,38236 &, 90978 & & 59 &, 150 \\
\hline AP-Alta & 31 & 13,7742 & 5,25807 &, 94438 & & & \\
AP-Baja & 30 & 12,4333 & 6,55314 & 1,19643 & & 59 &, 381 \\
\hline AT-Alta & 27 & 15,7407 & 6,04847 & 1,16403 & 3,339 & 59 &, 001 \\
AT-Baja & 34 & 11,0294 & 4,97561 &, 85331 & & & \\
\hline AG-Alta & 29 & 14,5172 & 6,13899 & 1,13998 & & & \\
AG-Baja & 32 & 11,8438 & 5,50137 &, 97251 & 1,794 & 59 &, 078 \\
\hline AE-Alta & 24 & 14,9583 & 6,52406 & 24 & & & \\
AE-Baja & 37 & 11,9189 & 5,24075 & 37 & 2,008 & 59 &, 049 \\
\hline APG-Alta & 27 & 12,8889 & 5,46551 & 1,05184 &,- 264 & 59 &, 793 \\
APG-Baja & 34 & 13,2941 & 6,33188 & 1,08591 & & & \multirow{2}{*}{, 106} \\
\hline ATE-Alta & 26 & 14,5385 & 6,43261 & 1,26154 & 1,642 & 59 & \\
\hline & & & & & & \\
\hline
\end{tabular}




\begin{tabular}{cccccccc} 
ATE-Baja & 35 & 12,0571 & 5,35747 &, 90558 & & & \\
\hline APGI-Alta & 31 & 13,7097 & 6,18166 & 1,11026 & \multirow{2}{*}{, 796} & 59 & \multirow{2}{*}{429} \\
APGI-Baja & 30 & 12,5000 & 5,67359 & 1,03585 & & & \\
\hline APGEX-Alta & 21 & 13,5714 & 5,66190 & 1,23553 & & & \\
APGEX-Baja & 40 & 12,8750 & 6,10669 &, 96555 & & 59 &, 666 \\
\hline ATEEX-Alta & 34 & 13,9118 & 6,15146 & 1,05497 & & \multirow{2}{*}{, 184} & \multirow{2}{*}{241} \\
ATEEX-Baja & 27 & 12,1111 & 5,56316 & 1,07063 & & & \\
\hline ATEI-Alta & 31 & 14,6452 & 6,24259 & 1,12120 & & & \\
ATEI-Baja & 30 & 11,5333 & 5,20433 &, 95018 & & 59 &, 039 \\
\hline
\end{tabular}

CREA-A = Lámina A del test CREA; ATOT = Atribuciones totales; AI = Atribuciones internas; AEX = Atribuciones externas; $\mathrm{AP}=$ Atribuciones permanentes; $\mathrm{AT}=$ Atribuciones temporales; $\mathrm{AG}=$ Atribuciones globales; $\mathrm{AE}=$ Atribuciones específicas $; \mathrm{APG}=$ Atribuciones permanentes y globales; $\mathrm{ATE}=$ Atribuciones temporales $\mathrm{y}$ específicas; APGI = Atribuciones permanentes, globales e internas; APGEX = Atribuciones permanentes, globales y externas; ATEEX = Atribuciones temporales, específicas y externas; ATEI = Atribuciones temporales, específicas e internas.

Tabla 6. Diferencias de medias en CREA-B según nivel alto o bajo en PDE. Media y desviación típica en CREA-B según nivel alto o bajo en PDE

\begin{tabular}{|c|c|c|c|c|c|c|c|}
\hline $\begin{array}{c}\text { Niveles de } \\
\text { PDE }\end{array}$ & $\mathrm{N}$ & Media & Dt & Error & $\mathrm{T}$ & g.l. & $\mathrm{P}$ \\
\hline ATOT-Alta & 28 & 16,0000 & 7,83629 & 1,48092 & \multirow{2}{*}{2,076} & \multirow{2}{*}{59} & \multirow{2}{*}{, 042} \\
\hline ATOT-Baja & 33 & 12,1212 & 6,75813 & 1,17644 & & & \\
\hline AI-Alta & 31 & 15,7097 & 7,07669 & 1,27101 & \multirow{2}{*}{1,967} & \multirow{2}{*}{59} & \multirow{2}{*}{, 054} \\
\hline AI-Baja & 30 & 12,0333 & 7,51772 & 1,37254 & & & \\
\hline AEX-Alta & 26 & 14,7692 & 8,23314 & 1,61465 & \multirow{2}{*}{, 779} & \multirow{2}{*}{59} & \multirow{2}{*}{,439 } \\
\hline AEX-Baja & 35 & 13,2571 & 6,89988 & 1,16629 & & & \\
\hline AP-Alta & 31 & 14,0645 & 6,44947 & 1,15836 & \multirow{2}{*}{, 172} & \multirow{2}{*}{59} & \multirow{2}{*}{,864 } \\
\hline AP-Baja & 30 & 13,7333 & 8,50531 & 1,55285 & & & \\
\hline AT-Alta & 27 & 17,3333 & 7,82009 & 1,50498 & \multirow{2}{*}{3,481} & \multirow{2}{*}{59} & \multirow{2}{*}{, 001} \\
\hline AT-Baja & 34 & 11,1765 & 5,99733 & 1,02853 & & & \\
\hline AG-Alta & 29 & 14,9310 & 6,79249 & 1,26133 & \multirow{2}{*}{1,025} & \multirow{2}{*}{59} & \multirow{2}{*}{, 309} \\
\hline AG-Baja & 32 & 12,9688 & 8,02610 & 1,41883 & & & \\
\hline AE-Alta & 24 & 16,4583 & 8,78724 & 1,79369 & \multirow{2}{*}{2,223} & \multirow{2}{*}{59} & \multirow{2}{*}{, 030} \\
\hline AE-Baja & 37 & 12,2432 & 6,03879 & ,99277 & & & \\
\hline APG-Alta & 27 & 13,2963 & 6,89378 & 1,32671 & \multirow{2}{*}{,- 561} & \multirow{2}{*}{59} & \multirow{2}{*}{, 577} \\
\hline APG-Baja & 34 & 14,3824 & 7,96589 & 1,36614 & & & \\
\hline
\end{tabular}




\begin{tabular}{|c|c|c|c|c|c|c|c|}
\hline ATE-Alta & 26 & 16,6538 & 8,36154 & 1,63983 & \multirow{2}{*}{2,596} & \multirow{2}{*}{59} & \multirow{2}{*}{, 012} \\
\hline ATE-Baja & 35 & 11,8571 & 6,07862 & 1,02747 & & & \\
\hline APGI-Alta & 31 & 14,3871 & 7,45957 & 1,33978 & \multirow{2}{*}{, 513} & \multirow{2}{*}{59} & \multirow{2}{*}{,610 } \\
\hline APGI-Baja & 30 & 13,4000 & 7,57309 & 1,38265 & & & \\
\hline APGEX-Alta & 21 & 14,0952 & 7,28632 & 1,59001 & \multirow{2}{*}{, 145} & \multirow{2}{*}{59} & \multirow{2}{*}{, 885} \\
\hline APGEX-Baja & 40 & 13,8000 & 7,65339 & 1,21011 & & & \\
\hline ATEEX-Alta & 34 & 14,8824 & 8,44147 & 1,44770 & \multirow{2}{*}{1,154} & \multirow{2}{*}{59} & \multirow{2}{*}{, 253} \\
\hline ATEEX-Baja & 27 & 12,6667 & 5,95496 & 1,14603 & & & \\
\hline ATEI-Alta & 31 & 15,6774 & 8,61157 & 1,54668 & \multirow{2}{*}{1,930} & \multirow{2}{*}{59} & \multirow{2}{*}{, 058} \\
\hline ATEI-Baja & 30 & 12,0667 & 5,64424 & 1,03049 & & & \\
\hline
\end{tabular}

CREA-B = Lámina B del test CREA; ATOT = Atribuciones totales; AI = Atribuciones internas; AEX = Atribuciones externas; $\mathrm{AP}=$ Atribuciones permanentes; $\mathrm{AT}=$ Atribuciones temporales; $\mathrm{AG}=$ Atribuciones globales; $\mathrm{AE}=$ Atribuciones específicas; $\mathrm{APG}=$ Atribuciones permanentes y globales; $\mathrm{ATE}=$ Atribuciones temporales $\mathrm{y}$ específicas; APGI = Atribuciones permanentes, globales e internas; APGEX = Atribuciones permanentes, globales y externas; ATEEX = Atribuciones temporales, específicas y externas; ATEI = Atribuciones temporales, específicas e internas.

\section{Discusión y conclusiones}

Los participantes que produjeron más explicaciones causales -en general- y más explicaciones causales resilientes -en particular-, ante hipotéticas adversidades, se asociaron con una mayor inteligencia creativa o producción divergente. En todos los cálculos sobresale la dimensión duración (una mayor producción de atribuciones temporales frente a hipotéticas adversidades), mostrando una clara relación con una mayor creatividad. Es importante señalar que la dimensión duración es la que más claramente ha demostrado ser un factor protector frente a la depresión (Seligman, 2005). También la dimensión amplitud (una mayor producción de atribuciones específicas frente a hipotéticas adversidades) en consonancia con la literatura científica como dimensión protectora de la depresión.

Respecto a la dimensión externa-interna, esta relación fue mayor en la dimensión Interna que en Externa a diferencia de lo que a nivel teórico podría esperarse según el modelo reformulado de indefensión aprendida (atribuciones temporales, específicas y externas), pero que iría en consonancia con lo hallado en otras investigaciones (Maldonado, Luque y Herrera, 1999). Por otra parte, este resultado estaría en la línea de la distinción que plantea Seligman et al (2005) entre creencias internas conductuales (por ejemplo, "tenía que haber estudiado 
más") y lo que llama creencias internas caracterológicas (por ejemplo, "soy un desastre como estudiante"). Las primeras son modificables y conducen a la acción de tratar de rectificar el problema por lo que impiden los sentimientos de desesperación e inutilidad. En cambio las segundas son permanentes dado que explican la ocurrencia de la adversidad por el carácter de la persona y llevan a sentimientos de incapacidad e inutilidad. Se teoriza, en base a los resultados, un posible efecto cascada o de retroalimentación, es decir, que la producción de explicaciones causales más resilientes (temporales y específicas) e internas ayudarán a producir estados de ánimo más positivos, así como una mayor sensación de dominio, lo que ayudará a producir mayor puntuación en la prueba de creatividad, mientras que las explicaciones causales más negativas producirían estados de ánimo más depresivos lo que, a nivel cuantitativo reduciría la puntuación en el test de creatividad. Whitmer y Gotlib (2013) señalan en su modelo de amplitud atencional, es posible que el estado de ánimo depresivo reduzca la atención y de ese modo disminuyen los repertorios de pensamiento y acción favoreciendo un pensamiento más repetitivo, creando así espirales emocionales negativas. Un mayor desarrollo del modelo teórico de los procesos psicológicos implicados sería una futura línea de trabajo. No se hallaron diferencias de género en las variables estudiadas.

\section{Implicaciones}

A nivel teórico supone un puente entre el modelo reformulado de indefensión aprendida como modelo de resiliencia y optimismo, con la inteligencia creativa. A nivel de evaluación psicológica aportaría un nuevo instrumento que enlaza las variables de pensamiento optimista con la producción divergente, muy relacionada con la creatividad en la línea de otras investigaciones que han tratado de ver la relación entre ambas variables (Sánchez-Hernández, Martín-Brufau, Méndez, Corbalán y Limiñana, 2010). La producción divergente explicativa podría relacionarse con modelos de solución de problemas (Guilford, 1977; Mahoney, 1981). Las explicaciones sobre las posibles causas que han producido un determinado problema podrían determinar el marco psicológico dentro del que se van a encontrar las posibles soluciones al mismo. La producción divergente explicativa (PDE) se contextualiza también dentro del constructo de flexibilidad psicológica, que entre otras características está relacionado con la capacidad de observar los problemas desde varios puntos de vista (Kashdan y Rottenberg, 2010). Esta nueva medida podría ayudar a ver el progreso de los participantes en el entrenamiento de esta habilidad en los programas de promoción del bienestar, de prevención de problemas psicológicos y/o en la intervención clínica. 
Una aplicación terapeútica, ya ideada por Sánchez-Hernández y Méndez (2009) inspirada en los estudios de Seligman et al. (2005) consiste en "El Sol Optimista". Consiste en dibujar un círculo y dentro de él se describe la adversidad. Luego el adolescente tiene que generar todas las posibles explicaciones por las que se puede haber producido la adversidad, siendo cada una de ellas un rayo de Sol. La hipótesis es que producir un mayor número de explicaciones ante acontecimientos negativos se relaciona con una mejor adaptación, ya que la persona contaría con más puntos de vista, tanto de causas internas sobre las que podría responsabilizarse e intervenir para encontrar la solución, como causas externas que le ayudarían a darle una visión más real de la situación y comprobar la existencia de otras causas que estarían fuera de su responsabilidad, lo que le ayudaría a comprender que no solo él/ella es causa directa del evento negativo, lo que protegería su autoestima. De igual forma, animar a producir atribuciones más contextuales y descriptivas de la situación concreta (temporales y específicas) en lugar de abstractas (permanentes y globales). También puede ser una prueba fácil de realizar en el inicio de la terapia ("dime todas las causas que se te ocurran de por qué te ha ocurrido dicha adversidad...") tanto para la evaluación como para el desarrollo de esta habilidad.

\section{Limitaciones y prospectivas}

Este trabajo puede considerarse un estudio piloto en la presentación de esta nueva variable y medida de flexibilidad psicológica teniendo como limitaciones el bajo tamaño muestral, la falta de un adecuado reclutamiento que garantice la representatividad de la muestra de la población estudiada, limitarse a estudiar la relación con la creatividad y por tanto la falta de otros datos psicométricos de la medidad de evaluación en su relación con otras variables , así como la necesidad de dar mayor profundidad al modelo teórico subyacente.

Siendo unos resultados interesantes, al relacionar el estilo explicativo resiliente con la creatividad, es necesario nuevos estudios con mayores muestras, estudiar la relación con otras variables afines que miden flexibilidad cognitiva, habilidad importante para una eficaz reestructuración cognitiva en terapia, (Johnco, Wuthrich y Rapee, 2014), flexibilidad explicativa (Fresco, Rytwinski y Craighead, 2006) y con otras medidas de creatividad. También sería interesante analizar la relación con el bienestar psicológico, depresión, autoconcepto, optimismo disposicional, curiosidad etc. Otra línea de investigación sería estudiar su papel en las hipótesis de los mediadores sobre la mejora en intervenciones preventivas y de tratamiento de 
problemas emocionales así como su relación con otras medidas que evalúan habilidades compensatorias siguiendo el trabajo de Barber y DeRubeis (1992).

En resumen este nuevo test y constructo refleja la relación entre dos conceptos clave, la resiliencia y la creatividad, por lo que sería importante continuar los estudios en esta nueva línea de investigación que cuenta con claras aplicaciones prácticas en la evaluación y tratamiento de los problemas emocionales, así como en la promoción del bienestar de la población.

\section{Referencias}

Abramson L. Y., Seligman M. E. P. y Teasdale J. (1978). Learned helplessness in humans: Critique and reformulation. Journal of Abnormal Psychology, 87, 49-74. doi:10.1037/0021-843X.87.1.49

Barber, J. P. y DeRubeis, R. J. (1992) The Ways of Responding: a scale to assess com pensa tory skills taught in cognitive therapy. Behavioral Assessment, 14, 93-115

Corbalán, F.J., Martínez, F., Donolo, D., Tejerina, M., Limiñana, R.M. (2003). CREA Inteli gencia Creativa. Una medida cognitiva de la creatividad. Madrid: TEA Ediciones. Obra galardonada con el VII Premio Internacional TEA Ediciones.

De Bono, E. (1998). El Pensamiento Lateral: Manual de Creatividad. Barcelona: Paidos Ibérica.

Fresco, D. M., Rytwinski, N. K. y Craighead, L. W. (2006). Explanatory flexibility and nega tive life events interact to predict depression symptoms. Journal of Social and Clinical Psychology, 26 (5), 595-608. doi: 10.1521/jscp.2007.26.5.595

Guilford, J.P. (1977). La naturaleza de la inteligencia humana. Buenos Aires, Paidos.

Hankin, B. L. y Abramson, L. Y. (2002). Measuring cognitive vulnerability to depression in adolescents: Reliability, validity, and gender differences. Journal of Clinical Child and Adolescent Psychology, 31, 491-504.doi: 10.1207/S15374424JCCP3104_8

Johnco, C., Wuthrich, V. M. y Rapee, R. M. (2014). The influence of cognitive flexibility on treatment outcome and cognitive restructuring skill acquisition during cognitive be havioural treatment for anxiety and depression in older adults: Results of a pilot study. Behaviour Research and Therapy 57, 55-64. doi: 10.1016/j.brat.2014.04.005

Kashdan, T. B. y Rottenberg, J. (2010). Psychological flexibility as a fundamental aspect of health. Clinical Psychology Review, 30, 865-878. doi: 10.1016/j.cpr.2010.03.001 
Mahoney, M. J. (1981). Ciencia personal: Una terapia cognitiva del aprendizaje. En A. Ellis y R. Grieger (eds.). Manual de terapia racional-emotiva (págs. 369-382). Bilbao, Declée de Brouwer

Maldonado, A., Luque, G. y Herrera, A. (1999). Cognición, estilo atribucional y depresión. Ansiedad y Estrés, 5 (2-3), 161-174.

Runco, M.A. (Ed). (1994). Problem finding, problem solving, and creativity. Creativity re search. Westport, CT, US: Ablex Publishing.

Sánchez-Hernández, Ó. y Méndez, F. X. (2009). Programa Sonrisa. Manuscrito no publi cado.

Sánchez-Hernández, O., Martín-Brufau, R., Méndez, F. X., Corbalan, F. J. y Limiñana, R.M. (2010). Relación entre optimismo, creatividad y síntomas psicopatológicos en estudiantes universitarios. Electronic Journal of Research in Educational Psychology, 8(3),1151-1178. Recuperado de http://www.redalyc.org/articulo.oa?id=293122000011

Schulman, P., Castellon, C. y Seligman, M. E. P (1989). Assessing explanatory style: the content analysis of verbatim explanationes and the attributional style questionnaire. Behaviour Research and Therapy, 27(5), 505-512.

doi: 10.1016/0005-7967(89)90084-3

Seligman, M.E.P. (1998). Aprenda optimismo: Haga de la vida una experiencia maravillosa. Barcelona: Grijalbo.

Seligman, M.E.P., Reivich, K., Jaycox, L. y Gillham, J. (2005). Niños optimistas. Barcelona: Random House Mondadori, S.A.

Torrance, E.P. (1974). The Torrance Tests of Creative Thinking-Norms-Technical Manual Research Edition-Verbal Tests, Forms A and B-Figural Tests, Forms A and B. Prince ton, NJ: Personnel Press.

Whitmer, A. J. y Gotlib, I. H. (2013). An attentional scope model of rumination. Psychologi cal Bulletin, 139, 1036-1061. doi: 10.1037/a0030923 